\title{
Gestión, liderazgo y valores en la administración de la Escuela de Física y Matemática de una IES del Ecuador
}

Management, leadership and values in the administration of the School of

Physics and Mathematics of an IES of Ecuador

Teresa Angélica Altamirano Novillo. ${ }^{1}$, Johanna Enith Aguilar Reyes. ${ }^{2}$, Fausto Francisco Navarrete Chávez. ${ }^{3}$ \& Verónica del Pilar Sánchez Alvear. ${ }^{4}$

\section{Recibido: 28-05-2019 / Revisado: 19-06-2019 /Aceptado: 30-07-2019/ Publicado: 06-09-2019}

\begin{abstract}
.
DOI: https://doi.org/10.33262/cienciadigital.v3i3.3.774

The quality of education and policy oriented to the training of directors of educational centers have been the most perceptive issues in Ecuadorian Higher Education.

The educational reforms of the last decades in Ecuador promote some changes for the administrators of the educational centers, in this case in the School of Physics and Mathematics, assigning them new responsibilities and complex demands in the administrative-financial management of the assigned resources.

Performing these important functions requires human talent with knowledge and preparation with a comprehensive education in science and values, more than enough reason that justifies the approach of the research topic "Management, Leadership and Values in the Administration of the School of Physics and Mathematics of the ESPOCH Faculty of Science”.

The project was carried out at the School of Physics and Mathematics, at the Faculty of Science of the Polytechnic School of Chimborazo.

Within this framework, the present investigation analyzed the management and leadership capacity integrated to the personal and institutional values that contribute to
\end{abstract}

1 Escuela Superior Politécnica de Chimborazo, Facultad de Ciencias. Riobamba, Ecuador, taltamirano@espoch.edu.ec

2 Escuela Superior Politécnica de Chimborazo, Facultad de Ciencias. Riobamba, Ecuador, johannae.aguilar@espoch.edu.ec

3 Escuela Superior Politécnica de Chimborazo, Facultad de Ciencias. Riobamba, Ecuador, fausto.navarrete@espoch.edu.ec

4 Escuela Superior Politécnica de Chimborazo, Facultad de Ciencias. Riobamba, Ecuador, v_sanchez@espoch.edu.ec 
raising the quality of education in higher level educational centers. As well as discovering a critical attitude to select, process and present valuable information that each of the authors of higher education experiences, determining the roles and leadership of managers in the execution of strategic and operational plans in the institution under investigation.

The research helped to know more about what management, leadership and values mean, and to know the current real situation and its vision in the individual, group and institutional progress of its actors.

Therefore, this institution constitutes an academic reference for current generations in ethical training and values.

Keywords: Administration, management, leadership, values, higher education.

\section{Resumen}

La calidad de la educación y la política orientadas a la formación de directivos de los centros educativos han sido los temas más perceptivos en la Educación Superior Ecuatoriana.

Las reformas educativas de las últimas décadas en el Ecuador promueven algunos cambios para los administradores de los centros educativos, en este caso en la escuela de Física y Matemática, asignándole nuevas responsabilidades y complejas demandas en el manejo administrativo-financiero de los recursos asignados.

Realizar estas importantes funciones requiere de talento humano con conocimientos y preparación con una formación integral en ciencia y valores, razón más que suficiente que justifica el planteamiento del tema de investigación "Gestión, Liderazgo y Valores en la Administración de la Escuela de Física y Matemática de la Facultad de Ciencias de la ESPOCH".

El proyecto se realizó en la Escuela de Física y Matemática, en la Facultad de Ciencias de la Escuela Superior Politécnica de Chimborazo.

Dentro de este marco la presente investigación, analizó la capacidad de gestión y liderazgo integrado a los valores personales e institucionales que permitan contribuir a elevar la calidad de la educación en los centros educativos de nivel superior. Así como descubrir una actitud crítica para seleccionar, procesar y presentar información valiosa que vivencia cada una de los autores de la educación superior, determinando los roles y liderazgo de los directivos en la ejecución de planes estratégicos y operativos en la institución investigada.

La investigación ayudó a conocer más sobre lo que significa gestión, liderazgo y valores, y conocer la situación real actual y su visión en el progreso individual, grupal e institucional de sus actores. 
Por lo tanto, dicha institución constituye un referente académico para las actuales generaciones en la formación ética y en valores.

Palabras claves: administración, gestión, liderazgo, valores, superior.

\section{Introducción.}

La administración debe basarse en valores. El verdadero liderazgo es, en el fondo, un dialogo sobre valores. El futuro de una institución se configura articulando valores, metáforas, símbolos y conceptos que orienten las actividades cotidianas de creación de valor por parte de sus miembros.

En otras palabras, hay que dar forma humanizada al propósito estratégico básico de la institución, en nuestro caso la calidad de la educación superior ecuatoriana.

De hecho, la administración de una institución educativa basada en valores debe lograr la generación de compromisos colectivos por proyectos nuevos e ilusionantes, para la mejora continua de la educación superior en el Ecuador.

\section{Marco Teórico}

\section{La gestión educativa: Concepto}

Acción y efecto de administrar (DRAE); Del latín gestĩo, el concepto de gestión hace referencia a la acción y al efecto de gestionar o de administrar. Gestionar es realizar diligencias conducentes al logro de un negocio o de un deseo cualquiera. Administrar, por otra parte, consiste en gobernar, dirigir, ordenar, disponer u organizar.

Es la capacidad de la institución para definir, alcanzar y evaluar sus propósitos, con el adecuado uso de los recursos disponibles.

El concepto de gestión posee preliminares básicos correspondientes a la organización, lo que implica que éste aplicado en una empresa o en un negocio examina algunos de los objetivos principales correspondientes a la misma.

Es importante tener en cuenta en este concepto que ninguna empresa puede subsistir si no posee algún tipo de beneficio, por lo que siempre se debe mantener un cierto nivel de competencia con las otras empresas que se dedican a fabricar y elaborar los mismos productos que la nuestra. El concepto de gestión se mueve en un ambiente en el cual, por lo general, los recursos disponibles escasean, y en base a esto, la persona encargada de la gestión debe aplicar diferentes fórmulas para que se esta manera se logre persuadir y motivar constantemente a todas las fuentes del capital empresarial, con el objetivo de lograr que las mismas apoyen todos los proyectos que la empresa planea emprender.

La gestión educativa como disciplina es relativamente joven; su evolución data de la década de los setenta en el Reino Unido y de los ochenta en América Latina. Desde entonces han surgido y se han desarrollado diversos modelos que representan formas de concebir la acción humana, los procesos sociales y el papel de los sujetos en el interior de éstos. 
Como punto de partida es importante enunciar el concepto de gestión educativa y establecer algunas diferencias que suelen hacerse entre gestión y administración. Aunque para la teoría de la administración, desde la década de los años sesenta, el concepto de gestión ha estado asociado con el término de gerencia y en especial, sobre el cómo gerenciar organizaciones, empresas productivas y de servicios, no ha sido así, para las instituciones del sector educativo.

Es importante aclarar que la gestión educativa busca aplicar los principios generales de la gestión, que han estado presentes en la teoría de la administración, al campo específico de la educación. El objeto de la gestión educativa como disciplina, es el estudio de la organización del trabajo en el campo de la educación, por tanto, está influenciada por teorías de la administración, pero, además, existen otras disciplinas que han permitido enriquecer el análisis, como son: la administración, la filosofía, las ciencias sociales, la psicología, la sociología y la antropología.

La acepción de gestión educativa está estrechamente relacionada con el concepto convencional de gestión administrativa, como una aproximación se presenta esta definición: La gestión educativa, se concibe como el conjunto de procesos, de toma de decisiones y realización de acciones que permiten llevar a cabo las prácticas pedagógicas, su ejecución y evaluación.

En vista de la marcada influencia que ejerce la administración sobre la gestión educativa, se ha trasladado las mismas escuelas del pensamiento administrativo, a la gestión educativa. Hoy en día, existe un consenso general que sostiene que la gestión educativa tiene tres grandes escuelas: la clásica, la de relaciones humanas y la de las ciencias del comportamiento administrativo.

\section{Importancia}

El saber de gestión es muy importante como recurso estratégico clave para la competitividad de una organización, en esta casa de una organización educativa. Para comenzar la gestión se comienza aclarando conceptos como datos, información y conocimiento que guiarán el resto de un proceso.

El directivo es quien se encuentra en la dirección del proceso para lograr la transformación de la institución a fin de que pueda ofrecer un servicio de excelencia. El proceso de dirección a ejercer y el conjunto de competencias requeridas para ello como, liderazgo, toma de decisiones y generación de un clima de participación y trabajo en equipo en la escuela, junto al instrumental metodológico requerido para lograrlo son básicos en cuanto a gestión.

La gestión es un proceso que comprende determinadas funciones y actividades laborales que los gestores deben llevar a cabo a fin de lograr los objetivos de la empresa. En la gestión, los directivos utilizan ciertos principios que les sirven de guía en este proceso. 
La gestión como disciplina, clasificar la gestión como una disciplina implica que se trata de un cuerpo acumulado de conocimientos susceptibles de aprendizaje mediante el estudio. Así pues, la gestión es una asignatura con principios, conceptos y teorías.

Estudiamos la gestión para entender esos principios, conceptos y teorías y para aprender la manera de aplicarlos en el proceso de la gestión empresarial. La gestión y las personas La palabra gestión, usada en este sentido, se refiere a las personas (gestores) que tienen a su cargo el proceso de gestión. Los gestores son las personas que asumen la responsabilidad principal por la realización del trabajo en una organización.

La gestión como carrera Los diferentes significados e interpretaciones del término gestión pueden interrelacionarse de este modo: las personas que quieren tener una carrera como gestores deberán estudiar la disciplina de gestión como medio para poner en práctica el proceso de gestión. Así, pues, definimos la gestión como el proceso emprendido por una o más personas para coordinar las actividades laborales de otras personas con la finalidad de lograr resultados de alta calidad que cualquier otra persona, trabajando sola, no podría alcanzar.

\section{La gestión educativa consiste en:}

- $\quad$ Presentar un perfil integral, coherente y unificado de decisiones.

- Definir los objetivos institucionales, las propuestas de acción y las prioridades en la administración de recursos.

- Definir acciones para extraer ventajas a futuro; se consideran tanto las oportunidades y amenazas del medio en el que está inserta, como los logros y problemas de la misma organización.

- Comprometer a todos los actores institucionales.

- Definir el tipo de servicio educativo que se ofrece. A su vez implica también:

- Participación de los profesores.

- Participación de padres.

- Administración del tiempo.

- Uso racional de los espacios.

- Movilizar recursos.

- Orientar recursos en una dirección en función de unos propósitos.

\section{Tipos de gestión}

Gestión de Conocimiento: se trata de un concepto aplicado en las organizaciones, que se refiere a la transferencia del conocimiento y de la experiencia existente entre sus miembros. De esta manera, ese acervo de conocimiento puede su utilizado como un recurso disponible para todos los miembros de la organización. 
Gestión Ambiente: es el conjunto de diligencias dedicadas al manejo del sistema ambiental en base al desarrollo sostenible. La gestión ambiental es la estrategia a través de la cual se organizan las actividades antrópicas que afectan el ambiente, con el objetivo de lograr una adecuada calidad de vida.

Gestión Administrativo: es uno de los temas más importantes a la hora de tener un negocio ya que de ella va depender el éxito o fracaso de la empresa. En los años hay mucha competencia por lo tanto hay que retroalimentarse en cuanto al tema.

Gestión Gerencial: es el conjunto de actividades orientadas a la producción de bienes (productos) o la prestación de servicios (actividades especializadas), dentro de organizaciones.

Gestión Financiera: se enfoca en la obtención y uso eficiente de los recursos financieros.

\section{Liderazgo educacional}

\section{Concepto de liderazgo}

Según el diccionario de la Real Academia el liderazgo es una situación de superioridad en que se halla una empresa, un producto o un sector económico, dentro de su ámbito.

Liderazgo, en psicología social, rol de la personalidad en el análisis de grupos pequeños. En sociología, influencia que se puede ejercer sobre una colectividad.

La corriente seguidora del alemán Max Weber, considerado el fundador de la sociología moderna, distingue tres tipos de liderazgo que se refieren a otras tantas formas de autoridad: el liderazgo carismático, al que sus seguidores le atribuyen condiciones y poderes superiores a los de otros dirigentes.

El liderazgo tradicional, que hereda el poder, ya sea por la costumbre de que ocupe un cargo destacado o porque pertenece a un grupo familiar que ha ostentado el poder desde hace mucho tiempo.

Y el liderazgo legal, que asciende al poder por los métodos oficiales, ya sean las elecciones o votaciones, o porque demuestra su calidad de experto sobre los demás. Esta figura se reconoce comúnmente en el campo de la política y de la empresa privada.

Es el proceso de ejercer una influencia mayor que lo que permite la estructura de dirección, más de lo que ella posibilita.

El liderazgo educacional se apoya en toda la teoría del liderazgo, pero debe asumir las características propias de su naturaleza y de su contenido.

El líder educacional es esencialmente un docente que debe dominar las funciones y tareas de cada puesto de trabajo, como una condición para el liderazgo efectivo, al demostrar su competencia profesional, y su interés profesional que es mejorar la educación, el cambio permanente del centro educacional, de acuerdo a las dimensiones de la tarea directiva educacional. 
Sobre esta base, tarea, contexto y fuerzas, el liderazgo educacional tiene que ser un fenómeno de equipo, ejercido por equipos de líderes.

El liderazgo educacional debe propiciar el desarrollo de todos sus subordinados, creando oportunidades, retirando barreras y obstáculos, y logrando una alta activación para propiciar el cambio, en primer lugar, en las personas.

Líder, es una persona que actúa como guía o jefe de grupo. Para que su liderazgo sea efectivo, el resto de los integrantes debe reconocer sus capacidades.

La sociología contemporánea subraya la importancia del papel que desempeña el poder que se le otorga y que ejerce el líder, o jefe, sobre los subordinados. También se interroga sobre cuál es la estructura que favorece que una persona se sitúe en posiciones superiores a las de los demás, cuál es la naturaleza de la legitimidad que obtiene el líder y de dónde proviene. En este supuesto conviene reflexionar sobre el papel de los medios de comunicación en los albores del siglo XXI.

El líder tiene la facultad de influir en otros sujetos. Su conducta o sus palabras logran incentivar a los miembros de un grupo para que trabajen en conjunto por un objetivo común. De acuerdo a su forma de ejercer la conducción del grupo, el líder puede ser considerado autoritario (toma las decisiones sin explicarlas ni justificarlas), democrático (discute con el grupo y decide por consenso) o laissez faire (es el líder liberal, aquel con una conducta pasiva que delega el poder en los demás)7.

El líder educacional es aquel que tiene un proyecto educativo, arrastra tras de sí a sus colaboradores y desarrolla a su personal.

\section{Características e importancia}

1. Es importante por ser la capacidad de un jefe para guiar y dirigir.

2. Una organización puede tener una planeación adecuada, control y procedimiento de organización y no sobrevivir a la falta de un líder apropiado.

3. Es vital para la supervivencia de cualquier negocio u organización.

4. Por lo contrario, muchas organizaciones con una planeación deficiente y malas técnicas de organización y control han sobrevivido debido a la presencia de un liderazgo dinámico.

El liderazgo está basado fundamentalmente en autoridad personal; Un líder puede ser definido como una persona capaz de unir a otro para el logro de un objetivo determinado o el arte de dirigir, coordinar y motivar individuos y grupos para que alcancen determinados fines; Consiste fundamentalmente en que el gerente líder posea buena capacidad expresiva, intelectual y social. 
ISSN: 2602-8085

www.cienciadigital.org

Vol. 3, N³.3, p. 111 - 133, septiembre, 2019

\section{Los valores y la educación}

¿Qué son los valores?

Uno de los temas más actuales y que son promovidos por toda clase de instituciones públicas y privadas, asociaciones, organizaciones no gubernamentales, partidos políticos, instituciones educativas, etc., es el tema de la cultura y de los valores. Esto obedece al hecho de que el hombre contemporáneo es consciente del deterioro que la cultura está teniendo. En efecto, el espíritu humano ha sufrido un tremendo deterioro de los valores que pueden sustentar la cultura.

El tema de los valores tiene una enorme vigencia. Se habla de la necesidad de que existan valores en la familia, en la escuela, en la sociedad. Las iniciativas sobre valores están presentes en los colegios, las empresas, medios de comunicación, etc. Y, aunque al mismo tiempo se reconozca constantemente la manifestación de conductas carentes de valores en todos los ámbitos y la sociedad sufra las consecuencias de la falta de valores en muchas circunstancias, a nivel mundial parece haber ansia de volver a los valores.

Se puede decir que la existencia de un valor es el resultado de la interpretación que hace el sujeto de la utilidad, deseo, importancia, interés, belleza del objeto. Es decir, la valía del objeto es en cierta medida, atribuida por el sujeto, en acuerdo a sus propios criterios e interpretación, producto de un aprendizaje, de una experiencia, la existencia de un ideal, e incluso de la noción de un orden natural que trasciende al sujeto en todo su ámbito.

Sócrates, estaba convencido que el conocimiento es la virtud más grande que puede poseer y practicar el ser humano. Para ello propone un ambicioso programa educativo cuyo camino es posible para unos "pocos privilegiados" pues el conocimiento moral involucra un estado de abstracción alto.

Los valores son el ser; están en él como rasgos que trascienden el ser mismo; son propiedades del ser. Dicho de otro modo, el valor se ancla en el ser y, como afirma W. Brugges, "anclar el valor en el ser significa justamente afianzar su carácter absoluto".

Tratándose del ser humano se observa que, al desarrollar y expresar su ser, su valor se matiza de diferentes formas, en función de las necesidades que satisface, de las actividades que realiza y de las facultades de su naturaleza que pone en juego y perfecciona.

De este modo, el ser del hombre se expresa de diferentes formas, en esferas de valor, es decir, en tipos de manifestaciones del valor de la persona humana, de acuerdo con los rasgos de su naturaleza, desde lo más corpóreo (o material) hasta lo más espiritual y, a partir de ello, al plano sobrenatural. (Marcela Chavarría Olarte, p.59).

Valores tales como honestidad, lealtad, identidad cultural, respeto, equidad, solidaridad, tolerancia, entre otros, son fundamentales para el convivir pacífico de la sociedad. 
Se entiende por valor moral todo aquello que lleve al hombre a defender y crecer en su dignidad de persona. El valor moral conduce al bien moral. Recordemos que bien es aquello que mejora, perfecciona, completa.

El valor moral perfecciona al hombre en cuanto a ser hombre, en su voluntad, en su libertad, en su razón. Se puede tener buena o mala salud, más o menos cultura, por ejemplo, pero esto no afecta directamente al ser hombre. Sin embargo, vivir en la mentira, el hacer uso de la violencia o el cometer un fraude, degradan a la persona, empeoran al ser humano, lo deshumanizan. Por el contrario, las acciones buenas, vivir la verdad, actuar con honestidad, el buscar la justicia, le perfeccionan. El valor moral te lleva a construirte como hombre, a hacerte más humano. Depende exclusivamente de la elección libre, el sujeto decide alcanzar dichos valores y esto sólo será posible basándose en esfuerzo y perseverancia. El hombre actúa como sujeto activo y no pasivo ante los valores morales, ya que se obtienen basándose en mérito.

Para lograr comprender plenamente los valores morales debemos analizar la relación que éstos guardan con otro tipo de valores. Siendo el ser humano el punto de referencia para los valores, cabe ordenarlos de acuerdo con su capacidad para perfeccionar al hombre. Un valor cobrará mayor importancia en cuanto logre perfeccionar al hombre en un aspecto más íntimamente humano:

a) Valores infrahumanos. - Son aquellos que sí perfeccionan al hombre, pero en aspectos más inferiores, en aspectos que comparte con otros seres, con los animales, por ejemplo. Aquí se encuentran valores como el placer, la fuerza, la agilidad, la salud.

b) Valores humanos inframorales. - Son aquellos valores que son exclusivos del hombre, ya no los alcanzan los animales, únicamente el hombre. Aquí encontramos valores como los económicos, la riqueza, el éxito, por ejemplo. La inteligencia y el conocimiento, el arte, el buen gusto. Y socialmente hablando, la prosperidad, el prestigio, la autoridad, etc.

c) Valores Instrumentales. - Son comportamientos alternativos mediante los cuales conseguimos los fines deseados.

d) Valores Terminales. - Son estados finales o metas en la vida que al individuo le gustaría conseguir a lo largo de su vida.

\section{Los valores y la educación}

Para los educadores los valores son un tema insoslayable; pugnar por "la educación en valores" no es una moda, sino la esencia misma de la acción educativa. La educación, o es "en valores" o no es tal, pues los valores son el contenido de la educación. 
Para educar en los valores hace falta conocerlos bien y habilitarse en la metodología apropiada para ello; es decir, partir del conocimiento firme de aspectos axiológicos básicos y, así, posteriormente, profundizar en directrices prácticas que permitan que la educación sea lo más auténtica e integral posible.

La educación en los valores es tarea de toda la vida. Nunca estamos suficientemente educados en algún valor, pues siempre podemos y aspiramos llegar a más. Es esto lo que da sentido a la vida humana: la búsqueda siempre inconclusa de la plenitud.

Como educadores debemos entender que la educación se opera en diferentes ámbitos, cada uno de los cuales tiene su responsabilidad específica en la integración de todos los valores para buscar el perfeccionamiento humano.

La familia es la primera escuela en valores tanto en formación moral y en la formación religiosa; después en el centro educativo se tiene o tenemos una responsabilidad ante la educación en determinadas áreas de valor. Un centro educativo es una institución de origen cultural, creado por la sociedad para apoyar y complementar la acción educativa familiar en aquellos aspectos que rebasan las posibilidades del microambiente familiar.

La educación en valores es sencillamente educar moralmente porque los valores enseñan al individuo a comportarse como hombre, a establecer jerarquías entre las cosas, a través de ellos llegan a la convicción de que algo importa o no importa, tiene por objetivo lograr nuevas formas de entender la vida, de construir la historia personal y colectiva, también se promueve el respeto a todos los valores y opciones.

La educación en valores es un proceso sistémico, pluridimensional, intencional e integrado que garantiza la formación y el desarrollo de la personalidad consciente; se concreta a través de lo curricular, extracurricular y en toda la vida universitaria. La forma de organización es el proyecto educativo.

La personalidad es "un sistema de alto nivel de integración de funciones síquicas del individuo, un complejo de formaciones estructuradas sobre ciertos principios que funcionan dirigidos a un objetivo" (D’Angelo, 1996).

Educar en valores es también educar al alumnado para que se oriente y sepa el valor real de las cosas; las personas implicadas creen que la vida tiene un sentido, reconocen y respetan la dignidad de todos los seres.

Los valores pueden ser realizados, descubiertos e incorporados por el ser humano, por ello reside su importancia pedagógica, esta incorporación, realización, descubrimiento son tres pilares básicos de toda tarea educativa; necesitan la participación de toda la comunidad educativa en forma coherente y efectiva.

La educación en valores debe contribuir a que el proyecto de vida se convierta en "un modelo de vida sobre la base de aquellas orientaciones de la personalidad que definen el sentido fundamental de su vida, y que adquieren una forma concreta de acuerdo con la construcción 
de un sistema de actividades instrumentadas, las que se vinculan con las posibilidades del individuo y, de otro lado las posibilidades objetivas de la realidad externa para la ejecución de esas orientaciones de la personalidad" (D'Angelo, 1996).

La educación en valores tiene como objetivo el alcance de una personalidad desarrollada o en desarrollo, la que se entiende, "al caracterizar a un individuo concreto donde el sistema de procesos y funciones que la forman se encuentran estructurados de manera armónica, en un proyecto de vida realista, donde predomina la autodirección consciente de los esfuerzos del individuo para lograr el desarrollo de sus potencialidades en forma creadora, así como su participación en la actividad social de acuerdo con valores de contenido progresista" (D’Angelo, 1996:4).

Educar en valores significa contribuir a la función integradora del individuo mediante la valoración de las contradicciones de la motivación, los intereses, etc.

La educación en valores debe coadyuvar a la tendencia interna de la personalidad a integrar y armonizar los factores internos y externos y a la autonomía de ésta, es decir, a la autorregulación sobre la base de fines conscientes, lo que está por supuesto, en interacción y en dependencia de la realidad social.

Los valores interiorizados conforman la esencia del modelo de representaciones personales, constituyen el contenido del sentido de vida, y de la concepción del mundo, permiten la comprensión, la interpretación y la valoración del sujeto y brindan la posibilidad de definir el proyecto de vida, integrado por objetivos y finalidades para la actividad social.

Otra peculiaridad de la educación en valores es su carácter intencional, consciente y de voluntad, no sólo por parte del educador, sino también del educando, quien debe asumir dicha influencia a partir de su cultura, y estar dispuesto al cambio. De ahí la importancia y la necesidad de conocer no sólo el modelo ideal de educación, sino las características del estudiante en cuanto a sus intereses, motivaciones, conocimientos, y actitudes, las que no están aisladas de las influencias del entorno ambiental.

Una comprensión clara de los límites objetivos del entorno, del modelo a que aspira la sociedad y de la subjetividad del estudiante permite dirigir mejor las acciones educativas y dar un correcto significado al contenido de los valores a desarrollar.

\section{Son tres las condiciones para la educación en valores:}

- Primera: conocer al estudiante en cuanto a: determinantes internas de la personalidad (intereses, valores, concepción del mundo, motivación, etc.); actitudes y proyecto de vida (lo que piensa, lo que desea, lo que dice y lo que hace).

- Segunda: conocer el entorno ambiental para determinar el contexto de actuación (posibilidades de hacer).

- Tercera: definir un modelo ideal de educación. 
"La educación en los valores es tarea de toda la vida. Nunca estamos suficientemente educados en algún valor, pues siempre podemos y aspiramos llegar a más, esto es lo que le da sentido a la vida humana: la búsqueda siempre inconclusa de la plenitud" (Chavarría Marcela, 2007)

Finalmente, lo fundamental para la educación en valores será entender cada vez más a fondo qué son los valores y cuáles son las esferas axiológicas en las que se manifiestan para el desarrollo humano, apuntar al perfeccionamiento integral del hombre como finalidad última, y perseverar en el ejemplo vivo como marco de referencia motivador y en la formación de la inteligencia y de la voluntad como instrumentos clave en la permanente conquista de la cima de cada una de las esferas de valor.

\section{Modelos de educación moral}

Estos tipos de modelos nacen por las interrogantes que se presentan por ejemplo el tema de la génesis de la moral. Entonces la formación de los sujetos no es única y surgen algunos modelos:

A. Modelos de transmisión de valores absolutos. - Comparten una idea heterónoma de la moral, es decir, una transmisión de valores inmodificables, válidos en cualquier situación, como impuestos, sin poder de elección de otra posibilidad. El individuo pierde autonomía.

B. Modelos de autoconocimiento y autenticidad moral. - Sucede cuando la educación moral tomada en este sentido se identifica con una concepción relativista de los valores, los valores absolutos entran en crisis, cada persona posee una escala de valores que le permite tomar decisiones.

A. Modelos de desarrollo de juicio moral. - Niegan la existencia de valores absolutos que deban transmitirse de generación a generación, pero tampoco comparte la creencia de que los conflictos morales únicamente puede solucionarse atendiendo a preferencias subjetivas, para ellos el papel de la educación debe centrarse en el desarrollo del juicio moral.

B. Modelos de socialización. - Considera la educación moral como socialización en tanto que pretenden insertar a los individuos en la colectividad a la que pertenecen, la sociedad es concebida como bien supremo del cual emana la moralidad, a las cuales todas las personas deben someterse aceptando las normas y valores que posibilitan y conforman la vida en sociedad.

C. Modelos de adquisición de hábitos morales. - Para este modelo una persona se considerará moral si su conducta la virtuosa, es decir, si realiza actos virtuosos y, además, los realiza de forma habitual y constante. 
D. Modelos de la construcción de la personalidad moral. - Parte de la idea de que la moral no es algo adquirido a priori, sino que se entiende como un producto cultural cuya creación depende de cada individuo y del conjunto de todos ellos.

\section{Valores en la escuela de física y matemática de la facultad de ciencias de la ESPOCH}

Se ha hablado del proceso de tecnificación y desarrollo de la ciencia y de la técnica al margen de los principios de la cultura llamado cientificismo o tecnocracia que significa que la ciencia y la técnica han sido mal utilizadas por el mismo hombre, que, al no tener una correcta escala de valores, ha quedado alienado y al servicio de estas. Esto se debe a que mientras las ciencias y las técnicas siguen su rumbo a un ritmo cada vez más acelerado, la cultura superior, estrictamente humana, la que perfecciona al hombre en cuanto hombre en sus dimensiones espirituales; la cultura de la inteligencia, de la voluntad y la libertad, de la ética, de la religión, de la política, de la administración y el derecho, de la verdadera comunicación, han estado decayendo hasta conducir al hombre a un alto grado de degradación personal y moral, de abusos contra la dignidad de la persona humana provocándose un caos político en el que el bien común y la verdad no son considerados.

Hoy en día se sostiene en universidades y centros educativos que la verdad es una construcción social, y que el bien es lo que la sociedad determine como bueno. La ambición y el deseo de poder ha cegado al hombre acerca de lo que él mismo es, ha perdido el dominio de sí mismo y ha perdido el verdadero sentido de su vida hasta el punto en que, bajo un aparente progreso y equilibrio, lo que se esconde es una decadencia y un caos político que cada vez es más difícil de ocultar. Precisamente por esto, por carecer de cultura espiritual, la cultura de lo material ha perdido el sentido humano haciendo que, aún en los países con más desarrollo económico y material no se hayan logrado mejorar los niveles de bienestar de la sociedad.

Es por esta razón que, en este breve estudio, he querido hacer una reflexión sobre los valores universitarios en la actualidad y su perspectiva hacia el futuro. Como punto de inicio es preciso dejar claro que para que sea una auténtica Universidad es necesario que se respete su esencia o naturaleza en la que la filosofía tiene un papel fundamental para educar en los valores y lograr un humanismo integral. Considero que hoy en día, las Universidades, lejos de restablecer el orden jerárquico de los distintos sectores de la cultura de acuerdo a una escala objetiva de valores, han sido las principales promotoras del desorden actual.

La Universidad debe reflexionar sobre su verdadera función social ya que debería ser el origen superior de la cultura de una nación con la misión de investigar la verdad en todos sus aspectos, pero sobre todo en la unidad superior de la sabiduría filosófica. 
Es por ello que la ESPOCH tiene como misión formar profesionales con una sólida base científica para incursionar en el campo de la física aplicada a los sistemas y procesos biológicos en instituciones y organismos nacionales e internacionales.

Es en la Escuela de física y matemática en donde se debe proyectar el orden y la organización de la vida humana, individual y social en todos sus aspectos; el cultivo o cultura de las facultades del hombre de la inteligencia para alcanzar la verdad total y de la voluntad para que la vida de todos se oriente, mediante la voluntad, a alcanzar esa verdad.

Es posible que la ESPOCH se especialice en uno u otro aspecto de la verdad, que cultive unas ciencias o técnicas en lugar de otras, pero, a lo que nunca puede renunciar es a su propia esencia, es decir, a la referencia e integración en la verdad absoluta de las verdades parciales a través de la filosofía ya que, de lo contrario, las ciencias y las técnicas no encontrarán su lugar para contribuir al bien propio del hombre para producir cultura.

En la Facultad de Ciencias, el profesor es el que se da para que el estudiante aprenda a pensar y por esta razón, el que ha de dirigir el movimiento de unificación de todos los saberes, es la parte vital del cuerpo, como el alma que integra la investigación, contemplación y la docencia ya que como ha quedado dicho, si falta uno de estos elementos no hay Universidad. Por otra parte, el estudiante es el que aprende a pensar, el que está en actividad constante, no porque se dedique a hablar o a realizar actividades técnicas sino porque aun cuando permanezca en silencio, existe una actividad interior que lo impulsa a seguir pensando y a seguir buscando y aún más a aportar algo al proceso.

\section{Metodología.}

La Escuela de Física y Matemática se caracteriza por tener una población estudiantil relativamente baja en relación con el resto de escuelas de la ESPOCH, se cuenta con un director de escuela, una secretaria y un conserje, la planta docente está compuesta por 24 personas entre nombramiento y contrato, la población estudiantil alcanza a 145 personas entre hombres y mujeres.

\section{Participantes}

Las personas involucradas en el proyecto son todos los integrantes de la Escuela de Física y Matemática, a quienes luego de conocer el universo vamos a evaluar por medio de encuestas.

\section{Materiales e instrumentos}

Los instrumentos de investigación son: encuesta dirigida a los profesores y alumnos, un cuestionario de entrevista para el director, las mismas están diseñadas para ser contestadas en base a su propia experiencia personal.

La entrevista puede cumplir diversas funciones: diagnóstica, orientadora, terapéutica, e investigadora. La ejecución de la entrevista va a comprender tres fases, 1) el contacto inicial 
con el entrevistado;2) la formulación de las preguntas; 3) la anotación de las respuestas.

No hay que olvidar que una buena entrevista debe reunir algunas características como, tener preguntas simples que reúnan requisitos de confiabilidad y validez. Así también el entrevistador debe poseer la suficiente agudeza para observar, escuchar, transcribir y sintetizar las respuestas del entrevistado, así como también debe tener una guía de entrevista en donde se establézcanlos objetivos y los aspectos más relevantes que se han de recopilar.

\section{Métodos}

Los métodos de investigación que aplicaremos son el descriptivo, analítico y sintético, que nos permitirá explicar y analizar el objeto de la investigación.

El método de investigación a realizar es de tipo exploratorio y descriptivo, ya que facilitará explicar y caracterizar la realidad de la gestión en el liderazgo educativo y la promoción de valores en la escuela de Física y Matemática, de tal manera, que haga posible conocer el problema en estudio tal cual se presenta en la realidad. Entonces este tipo de investigación facilita acceder al conocimiento de la realidad para plantear el problema, declarar las hipótesis, aclarar conceptos, reunir información y familiarizarse con el proceso del conocimiento del problema en estudio.

En el proceso de investigación se requerirá también el método analítico - sintético, pues facilitará la desestructuración del objeto de estudio en todas sus partes y la explicación de las relaciones entre elementos y el todo, así como también la reconstrucción de las partes para alcanzar una visión de unidad, asociando juicios de valor, abstracciones, conceptos que ayudarán a la comprensión y conocimiento de la realidad.

El método inductivo y el deductivo a utilizarse permitirá configurar el conocimiento y a generalizar de forma lógica los datos empíricos a alcanzarse en el proceso de investigación.

El método estadístico hará factible organizar la información alcanzada, facilitará los procesos de validez y confiabilidad de los resultados y, finalmente tendrá su aplicación práctica en el proceso de la verificación de las hipótesis.

El método hermenéutico, permitirá la recolección e interpretación bibliográfica en la elaboración del marco teórico y, además, facilitará el análisis de la información empírica a la luz del marco teórico.

Para la recolección y análisis de la información teórica y empírica, se utilizarán las siguientes técnicas e instrumentos de investigación:

La Lectura. - como medio importante para conocer, analizar y seleccionar aportes teóricos, 
conceptuales y metodológicos sobre liderazgo y valores.

Los mapas conceptuales y organizadores gráficos. - como medios para facilitar los procesos de comprensión y síntesis de los apoyos teórico - conceptuales.

El Cuestionario. - se utilizará para le recuperación de la información de campo. Servirá para obtener información sobre las variables y de esta manera construir el diagnóstico sobre liderazgo educativo y valores.

\section{Resultados}

\section{Diagnóstico}

La gestión en liderazgo y valores es importante dentro de una institución educativa, de esta manera se consigue lograr los objetivos propuestos y encaminar a la institución al reconocimiento en su actividad, la Escuela de Física y Matemática se rige al reglamento interno de la ESPOCH y cumple con la misión y visión de la institución. Siendo este el punto de partida se enlistan a continuación los instrumentos de gestión educativa en donde se evidencia la gestión el liderazgo y valores dentro de la Escuela de Física y Matemática de la Facultad de Ciencias de la ESPOCH.

- Manual de organización

- $\quad$ Código de ética

- Plan estratégico

- Plan operativo anual

- $\quad$ Proyecto educativo institucional

- $\quad$ Reglamento de régimen académico

\section{Estructura organizativa de la institución}

La realidad institucional, señala que debemos asumir una actitud positiva frente a los grandes desafíos, mejorar ante el estancamiento y la apatía, implementar una cultura organizativa, con coordinación y responsabilidad, entendiendo que la estructura de una organización es simplemente la suma total de las formas en que su trabajo es dividido en diferentes tareas con una adecuada coordinación entre ellas, para el cumplimiento efectivo de su misión, procurando que la Escuela de Física y Matemática se constituya en un verdadero sistema abierto y dinámico, que implique integridad y unificación para lograr el funcionamiento óptimo del conjunto de sus componentes.

\section{Dimensión pedagógica curricular y valores}

En este apartado se realiza un proceso colectivo de análisis reflexión, discusión y recolección de información sobre las actividades escolares que forman parte de la gestión pedagógica de la escuela, es un espacio para construir competencias de lo que el Director Escolar y Docentes hacen para entender y mejorar los procesos de enseñanza. Sin dejar de lado los 
valores institucionales.

- Dimensión organizativa operacional y valores

- Dimensión administrativa y financiera y valores

- Dimensión comunitaria y valores

\section{Proceso de aplicación de los instrumentos de investigación}

El proceso de aplicación de los instrumentos se lo realiza en la Escuela de Física y Matemática, considerando a todos los protagonistas dentro de este proyecto; tenemos una encuesta para los directivos, en este caso para el director de la Escuela, otra encuesta fue aplicada a los docentes que constituyen la muestra total 24 personas, y una encuesta aplicada a los estudiantes que son un total de 20 los que constituyen la muestra.

Por otro lado, se aplica un cuestionario de entrevista la información suministrada a través de este medio, servirá como aporte para fundamentar su informe de tesis y para potenciar la propuesta de innovación para la Gestión de la Organización sustentada en valores y liderazgo, la misma será aplicada a los directivos: Rector, director, Supervisor.

Y como es una institución de educación superior, las encuestas para los padres de familia no fueron realizadas.

\section{Gráficos de los resultados}

Figura 1. El rol del Docente Líder Se Define como una persona que posee la actitud.

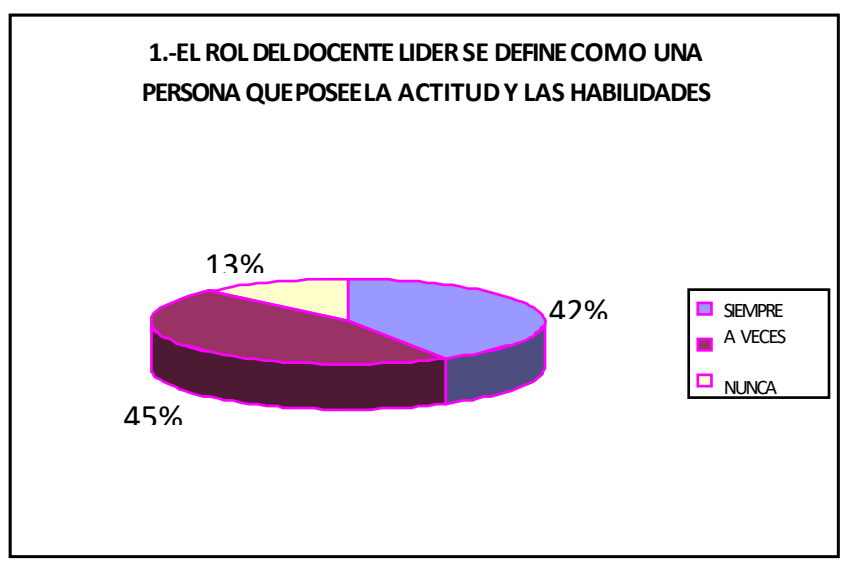

Fuente: Encuestas aplicadas a docentes de la escuela de Física y Matemática

Se observa que el $45 \%$ de los profesores piensan que a veces el rol del docente líder se define como una persona que posee la actitud y las habilidades para cuestionar las órdenes existentes; mientras que el $42 \%$ piensa que siempre el rol del docente líder se define como una persona que posee la actitud y las habilidades para cuestionar las órdenes existentes; y el $13 \%$ piensa que nunca el rol del docente líder se define como una persona que posee la actitud y las habilidades para cuestionar las órdenes existentes. 
Figura 2. El Liderazgo

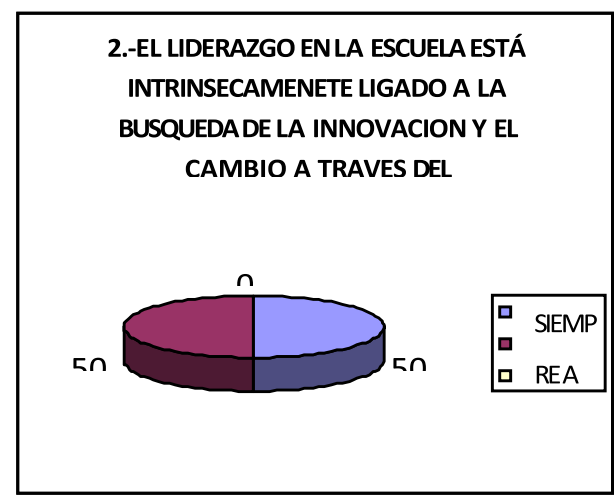

Fuente: encuestas aplicadas a docentes de la escuela de Física y Matemática

Se demuestra que el $50 \%$ de docentes piensa que siempre y el otro $50 \%$ piensa que a veces el liderazgo en la escuela está intrínsecamente ligado a la búsqueda de la innovación y el cambio a través del cuestionamiento constante de transformar las formas habituales de enseñanza.

Figura 3. Los Directivos y Docentes

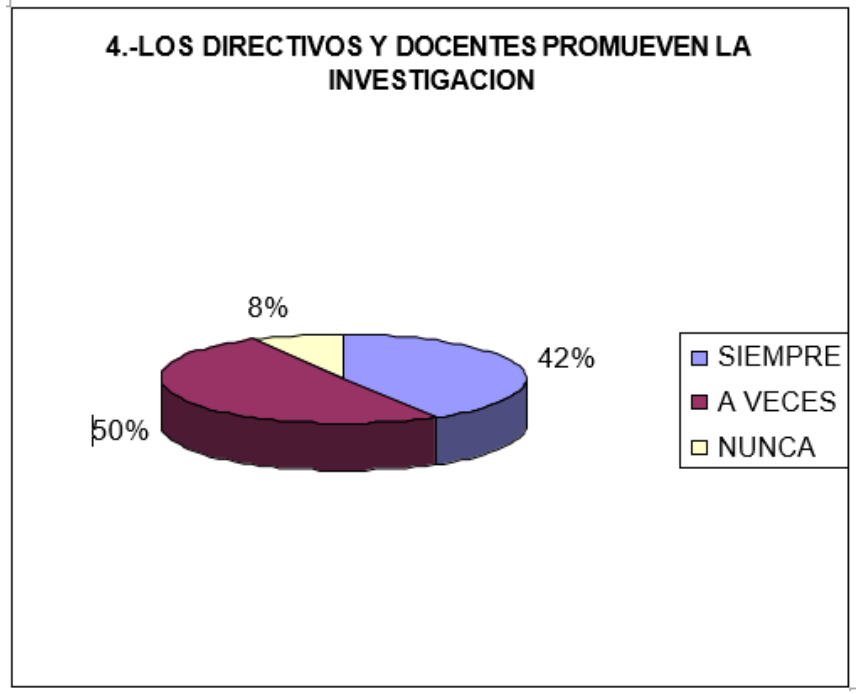

Fuente: encuestas aplicadas a docentes de la escuela de Física y Matemática

Los directivos y docentes a veces promueven la investigación; el $42 \%$ dice que los directivos y docentes promueven la investigación; y sólo el $8 \%$ dice que nunca los directivos y docentes promueven la investigación. 
Figura 4. Trabajo en Equipo

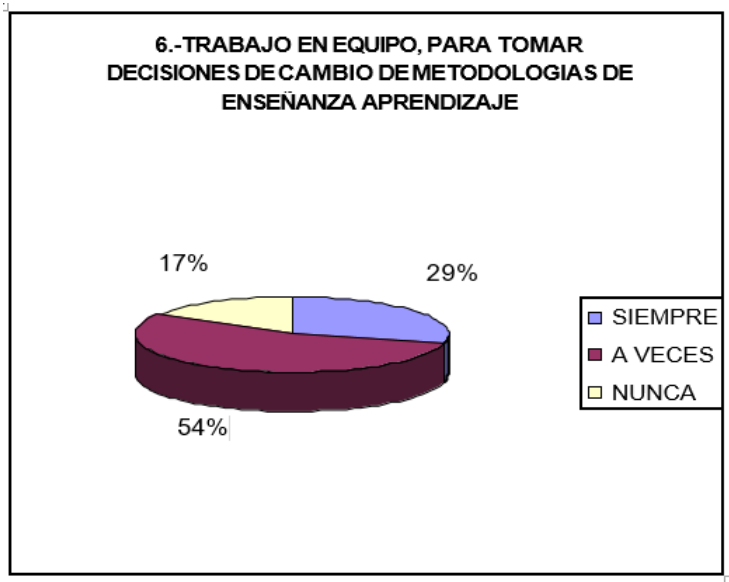

Fuente: encuestas aplicadas a docentes de la escuela de Física y Matemática

La figura 4 muestra que el $54 \%$ de los docentes dice que a veces trabaja en equipo para tomar decisiones de cambio de metodologías de enseñanza-aprendizaje; el 29\% dice que siempre trabaja en equipo para tomar decisiones de cambio de metodologías de enseñanzaaprendizaje; el 17\% dice que nunca trabaja en equipo para tomar decisiones de cambio de metodologías de enseñanza-aprendizaje.

Figura 5. Proceso de Enseñanza

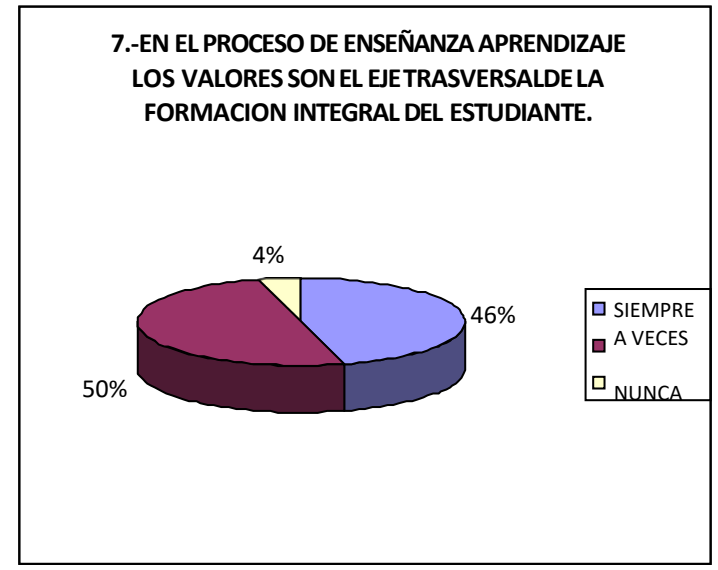

Fuente: encuestas aplicadas a docentes de la escuela de Física y Matemática

En la figura 5 tenemos que los docentes dicen que el $50 \%$ a veces, el $46 \%$ dice que siempre, y el $4 \%$ dice que nunca en el proceso de enseñanza-aprendizaje los valores son el eje trasversal de la formación integral del estudiante. 
Figura 6. Desacuerdo Continuo

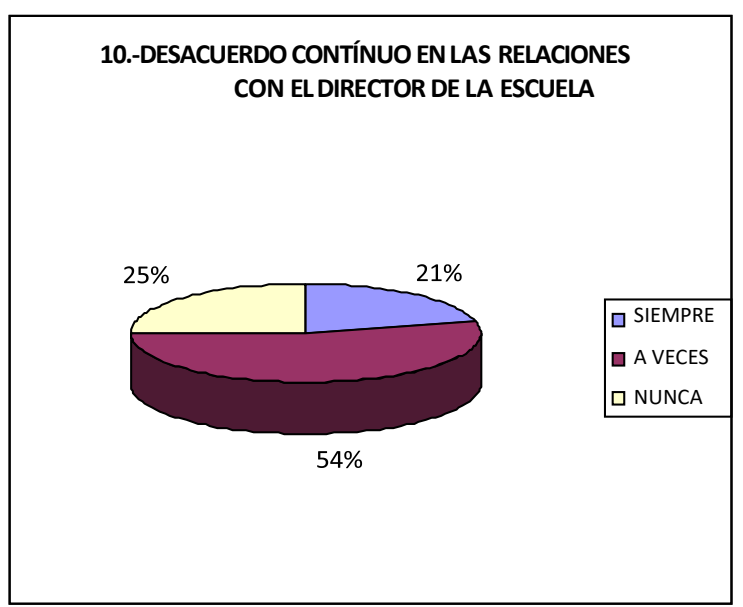

Fuente: encuestas aplicadas a docentes de la escuela de Física y Matemática

La figura 6 enseña que el 54\% de los docentes dice que a veces hay desacuerdo continuo en las relaciones con el director de la escuela; el $25 \%$ die que nunca hay desacuerdo continuo en las relaciones con el director de la escuela; y el $21 \%$ dice que siempre hay desacuerdo continuo en las relaciones con el director de la escuela.

Figura 7. Los Directivos

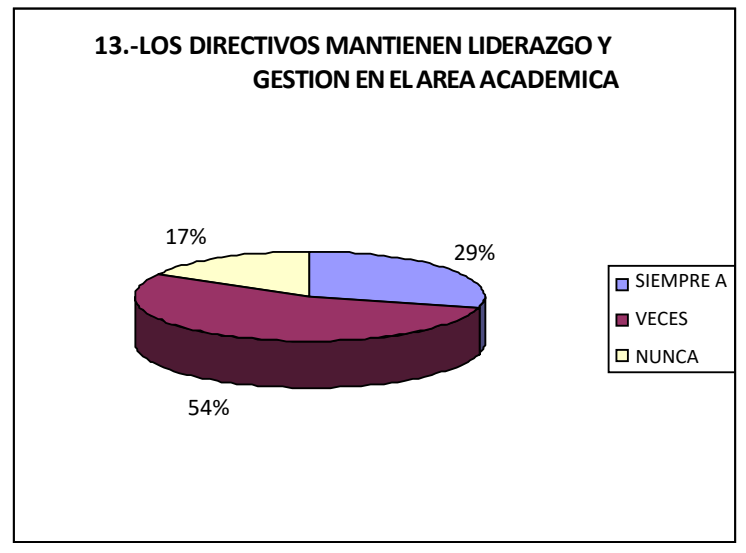

Fuente: encuestas aplicadas a docentes de la escuela de Física y Matemática

La figura 7 indica que el $54 \%$ de los docentes dice que a veces los directivos mantienen liderazgo y gestión en el área académica; el 29\% de los docentes dice que siempre los directivos mantienen liderazgo y gestión en el área académica; y el 17\% de los docentes dicen que nunca los directivos mantienen liderazgo y gestión en el área académica. 
Figura 8. Los Directivos y el liderazgo

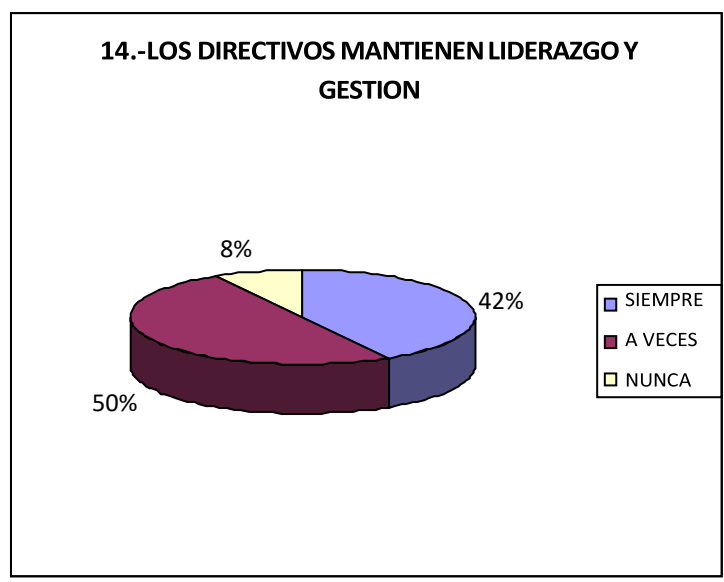

Fuente: encuestas aplicadas a docentes de la escuela de Física y Matemática

La figura 8 muestra que el 50\% de los docentes dicen que a veces los directivos mantienen liderazgo y gestión en el área administrativa-financiera; el $42 \%$ de los docentes dice que siempre los directivos mantienen liderazgo y gestión en el área administrativa-financiera; y el 8\% de los docentes dice que nunca los directivos mantienen liderazgo y gestión en el área administrativa-financiera.

Figura 9. Los Valores

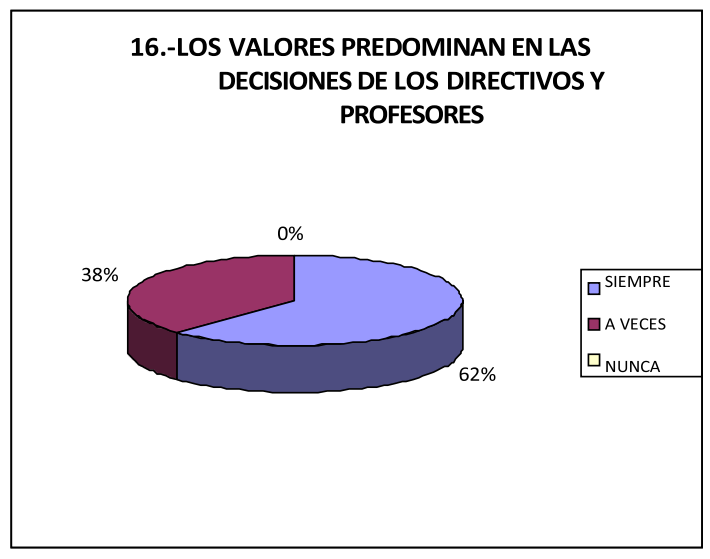

Fuente: encuestas aplicadas a docentes de la escuela de Física y Matemática

En la figura 9 se aprecia que el $62 \%$ de los docentes dice que siempre los valores predominan en las decisiones de los directivos y profesores; el 38\% de los docentes dice que a veces los valores predominan en las decisiones de los directivos y profesores; mientras que el $0 \%$ dice que nunca los valores predominan en las decisiones de los directivos y profesores. 


\section{Conclusiones}

- Los equipos de trabajo en la escuela están dirigidos por los coordinadores de área, en el caso de la Escuela de Física y Matemática hay las áreas básicas y las de ejercicio profesional, esto ayuda a trabajar en conjunto con todos.

- Las tareas de los miembros de la escuela se encuentran escritas en el manual de normas, reglas y procedimientos, es decir en el estatuto politécnico descrito anteriormente.

- El clima de respeto y consenso en la toma de decisiones está liderado por el director, quien es el líder y toma un papel muy importante para poder desarrollar todas las actividades establecidas.

- Para la resolución de conflictos y promover soluciones pertinentes y oportunas en el tiempo, el director delega la toma de decisiones a un grupo de colaboradores y este grupo está integrado por docentes y estudiantes lo que significa que si hay una organización dentro de la escuela.

\section{Referencias bibliográficas.}

Bennis, Warren y Burt Nanus, (1985). Líderes: el arte de mandar. México: Merlín Libros. Brugges, Walter. (1988). Diccionario de filosofía. Barcelona: Herder.

Buele Maldonado, Mariana. (2011). Instructivo para la elaboración de tesis, proyecto de grado II. Loja Ecuador.

CAMARGO, Marina y DUSSÁN, Marina (2002). Investigación Educativa e Innovación Pedagógica. Colombia Ciencia y Tecnología - COLCIENCIA. Bogotá.

Chavarría Olarte, Marcela. (2007). Educación en un mundo globalizado. México: Editorial Trillas.

D’ Angelo, O. (1996). Autorrealización de la personalidad. La Habana: Ed. Academia.

Fipella, Yaime y Ramón Pes Puig. (1987). "Liderazgo Transformacional", Revista "Alta Dirección", No. 133.

González, Juan Carlos. (1988). "El Liderazgo, un punto de vista para su estudio". La Habana: CETDIR

LATAPI, Pablo (2000). Valores y educación. México: UNAM.

MOORE, T. W. (1996). Educación en valores. Madrid. Grupo Anaya Editorial. Primera edición.

Sayles, Leonard R. (2011). "Liderazgo". México: Mc Graw-Hill.

TORRES, Mercedes. (2001). “Dirección, liderazgo y gestión escolar”. México: La Nueva escuela II.

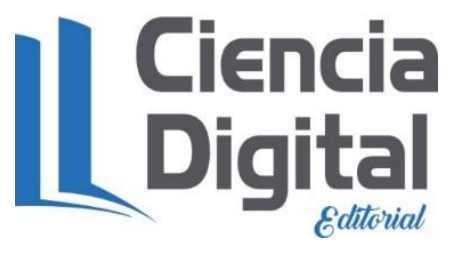




\section{PARA CITAR EL ARTÍCULO INDEXADO.}

Altamirano Novillo, T., Aguilar Reyes, J., Navarrete Chávez, F., \& Sánchez Alvear, V. del P. (2019). Gestión, liderazgo y valores en la administración de la Escuela de Física y Matemática de una IES del Ecuador. Ciencia Digital, 3(3.3), 111-133. https://doi.org/10.33262/cienciadigital.v3i3.3.774

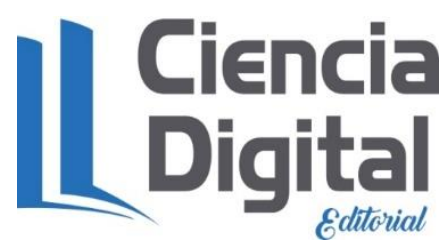

El artículo que se publica es de exclusiva responsabilidad de los autores y no necesariamente reflejan el pensamiento de la Revista Ciencia Digital.

El artículo queda en propiedad de la revista y, por tanto, su publicación parcial y/o total en otro medio tiene que ser autorizado por el director de la Revista Ciencia Digital.
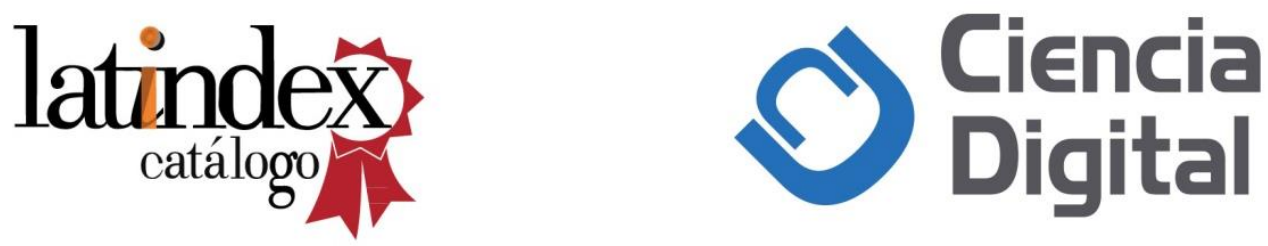